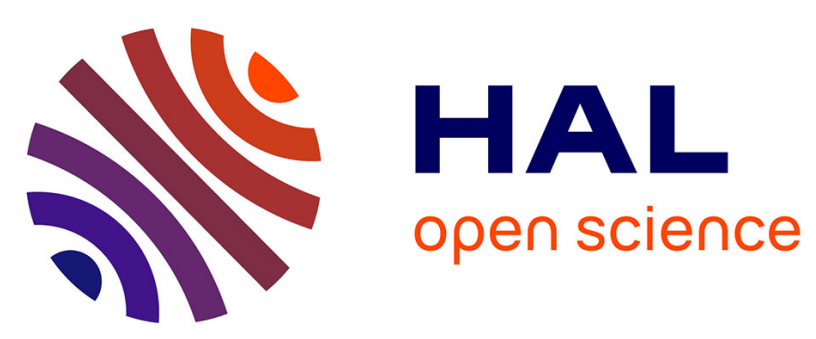

\title{
Physical properties of ternary organic conductors of TCNQ and iodine: role of the counterions in the low temperature instabilities
}

C. Coulon, P. Delhaes, S. Flandrois, J. Amiell, E. Bonjour, Pascal Dupuis

\section{To cite this version:}

C. Coulon, P. Delhaes, S. Flandrois, J. Amiell, E. Bonjour, et al.. Physical properties of ternary organic conductors of TCNQ and iodine: role of the counterions in the low temperature instabilities. Journal de Physique, 1985, 46 (5), pp.783-792. 10.1051/jphys:01985004605078300 . jpa-00210020

\section{HAL Id: jpa-00210020 https://hal.science/jpa-00210020}

Submitted on 1 Jan 1985

HAL is a multi-disciplinary open access archive for the deposit and dissemination of scientific research documents, whether they are published or not. The documents may come from teaching and research institutions in France or abroad, or from public or private research centers.
L'archive ouverte pluridisciplinaire HAL, est destinée au dépôt et à la diffusion de documents scientifiques de niveau recherche, publiés ou non, émanant des établissements d'enseignement et de recherche français ou étrangers, des laboratoires publics ou privés. 


\title{
Physical properties of ternary organic conductors of TCNQ and iodine : role of the counterions in the low temperature instabilities
}

\author{
C. Coulon $\left({ }^{+}\right)$, P. Delhaes $\left({ }^{+}\right)$, S. Flandrois $\left({ }^{+}\right)$, J. Amiell $\left({ }^{+}\right)$, E. Bonjour $\left({ }^{++}\right)$and P. Dupuis $\left({ }^{+++}\right)$ \\ $\left({ }^{+}\right)$Centre de Recherche Paul Pascal, Domaine Universitaire, 33405 Talence, France \\ $\left({ }^{++}\right)$Centre d'Etudes nucléaires de Grenoble, Service des basses températures, 85X, \\ 38041 Grenoble Cedex, France \\ $\left({ }^{++}\right)$Laboratoire de Chimie Physique macromoléculaire ENSIC, INSN, 1, rue Grandville, \\ 54000 Nancy Cedex, France
}

(Reçu le 24 avril 1984, révisé le 26 novembre, accepté le 3 janvier 1985)

\begin{abstract}
Résumé. - Plusieurs propriétés physiques des sels d'anions radicaux d'une série de composés ternaires, comprenant le TCNQ, l'iode et un sel d'ammonium quaternaire, ont été étudiées. Plusieurs transitions de phase ont été détectées à basse température. A partir d'une étude structurale récente sur un de ces sels, le rôle des contre-ions est discuté afin d'expliquer les différents comportements observés dans cette série de composés. Finalement une description unifiée de la série incluant à la fois les interactions entre chaînes de TCNQ et les deux contre-ions présents est proposée.
\end{abstract}

\begin{abstract}
Various physical properties of several radical-anion salts of a series of ternary compounds made with TCNQ, iodine and quaternary ammonium are described. Several phase transitions are found at low temperature. Following a recent structural study of one of these salts, the role of the counterions is discussed to explain the different behaviours found in the series. Finally, a unified description of the series involving both the interactions between the TCNQ chains and with the two counterions (iodine and ammonium) is presented.
\end{abstract}

\section{Introduction.}

The study of " one chain " organic conductors has been recently renewed with the discovery of superconducting properties in several of these compounds [1], radical-cation salts issued from tetrathiafulvalene (TTF) derivatives. At the opposite end of the spectrum, a former class of ion radical salts based on tetracyanoquinodimethane (TCNQ), which is a strong electron acceptor, has been known for quite some time. Among them, a peculiar series of ternary compounds, made with both iodine and various quaternary ammonium cations as counterions, is particularly interesting [2]. Changing the size of the alkyl ammonium has allowed us to synthesize a series of isomorphous and isostructural compounds (as far as the structure of the radical anion lattice is concerned). Only the unit cell parameter which involves the ammonium group (the $c$-axis, as described later on) is noticeably changed, and a comparison of the physical properties for the different salts indicates the role of the interstack couplings [3] : we have shown that the temperature of the metal-insulator transition is a decreasing function of the cation size. In this comparative study the role of the two counterions has not been considered in detail. However, a recent study on the basic compound of this series (the trimethylammonium-TMA-salt) clearly shows that the role of these counterions has to be considered [4]. The order of the iodine sublattice is rather important because the $\mathrm{I}_{3}^{-}$ chains are shown to be involved in two of the three phase transitions which have been observed for this salt. Moreover, for a few compounds with isoalkyl ammonium groups, the temperature of the metalinsulator transition has been found to be too high when compared with the calculated electrostatic interchain coupling $[3,5]$. For these reasons, new experiments on these series of compounds were necessary in order to understand the role of the two counterions : this is the purpose of this paper.

The preparation of the compounds has already been described [3]. A well defined stoichiometry is obtained for both monocations and dications. The corresponding raw formulas read : $\mathbf{M}^{+}$-TCNQ-I where $\mathbf{M}^{+}$is a monocation (with an alkyl or isoalkyl chain) and $\mathrm{M}^{2+}-\mathrm{TCNQ}_{2}-\mathrm{I}_{2}$ where $\mathrm{M}^{2+}$ is a dication. 
A large number of cations can be used. However, when the size of the cation is increased, only poor quality crystals can be prepared preventing any detailed analysis of the salts. In this paper we will be only interested in the four typical compounds for which very nice crystals were obtained. We will present new experimental results allowing a more complete discussion which clearly shows the role played by the counterions. The salts will be labelled using an acronym given in table $I$ in which the chemical formula of the cations is also given. After a brief structural presentation of the series, these new experimental results will be given. The last part of the paper will be devoted to the discussion of the observed properties and the concluding remarks.

\section{Survey of the main structural properties of these salts.}

1.1 Crystal structures at $295 \mathrm{~K}$. - The room temperature structural properties of the TMA salts have been extensively studied. The organic lattice, which presents a monoclinic structure with the space group $\mathrm{C} 2 / \mathrm{m}$, is composed of TCNQ chains, with a zig-zag stacking along the $b$ axis, between which are intercalated the cations in the $c$ direction; the TMA ions are interacting with the TCNQ molecules through hydrogen bonds (see Fig. 1a). The iodine arrays, organized in $\mathrm{I}_{3}^{-}$chains, alternate with the TCNQ stacks along the $a$ axis. In most of the crystals a long-range order is found in the $a$ and $c$ transverse directions between the iodine chains even if a small amount of disorder (probably due to defects in the stacking of the
$\mathrm{I}_{3}^{-}$chains) is always visible. A few crystals were also prepared with the same organization of the organic lattice but with a disordered $\mathrm{I}_{3}^{-}$sublattice. They will be called disordered (D) crystals in the following. However most of the crystals are ordered $(\mathrm{O})$ crystals for which the room temperature long range order was determined [7]. Although a complete description of this organization requires several Fourier components we will approximate this charge distribution in the following using a periodic distribution with the iodine wave vector $q_{\mathrm{I}}=\left(2 / 3 a^{*}, 2 / 3 b^{*}, c^{*}\right)$ where $a^{*}, b^{*}, c^{*}$ are the reciprocal wave vectors of the organic lattice at room temperature. This approximation has been shown to be relevant for the description of the low temperature properties of TMA-TCNQ-I [4]. Furthermore, for every crystal of appreciable size two sorts of macroscopic domains are seen corresponding to opposite $a^{*}$ components of $q_{\mathrm{r}}$.

Most of the other salts of the series have a structural organization at $300 \mathrm{~K}$ close to that of the TMA crystals as far as the organic lattice is concerned [3]. For example, figure $1 \mathrm{~b}$ shows the room temperature structure of TMHDA-TCNQ $-\mathrm{I}_{2}$. Because of the presence of a dication, one TMHDA is interacting with two different TCNQ molecules belonging to two neighbouring chains. For this compound the structure of the iodine sublattice is not precisely known. However, preliminary X-ray measurements indicate that this salt also has a domain-like iodine structure. The corresponding components of the iodine wave vectors $q_{\mathrm{I}}$ will also have non integer values if expressed using the

Table I. - List of cations, acronyms used and temperatures of the observed phase transitions. $T_{\mathrm{c} 1}$ is determined from d.c. electrical resistivity or magnetic susceptibility measurements and $X$-ray data. $T_{\mathrm{c} 2}$ and $T_{\mathrm{c} 3}$ are detected by specific heat or EPR experiments and X-ray data.

\begin{tabular}{|c|c|c|c|c|}
\hline Cation & Acronym & $\begin{array}{c}T_{\mathrm{c} 1}(\mathrm{~K}) \\
\text { metal.- } \\
\text { insulator }\end{array}$ & $\begin{array}{c}T_{\mathrm{c} 2}(\mathrm{~K}) \\
\text { structural }\end{array}$ & $\begin{array}{c}T_{\mathrm{c} 3}(\mathrm{~K}) \\
\text { comm.-incom- } \\
\text { mensurate }\end{array}$ \\
\hline 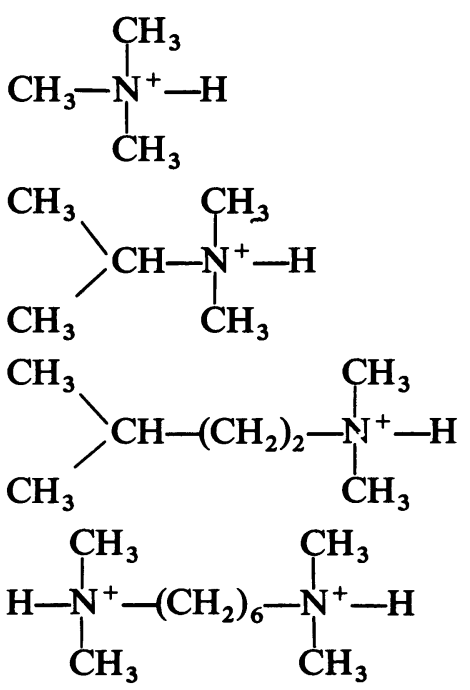 & $\begin{array}{l}\text { TMA crystals } \\
\text { IPrDMA } \\
\text { (O) crystals } \\
\text { IPentDMA } \\
\text { TMHDA }\end{array}$ & $\begin{array}{l}- \\
150 \\
139\end{array}$ & $\begin{array}{c}- \\
\sim 85 \\
(60-100) ?\end{array}$ & $\begin{array}{r}65 \\
-\end{array}$ \\
\hline
\end{tabular}




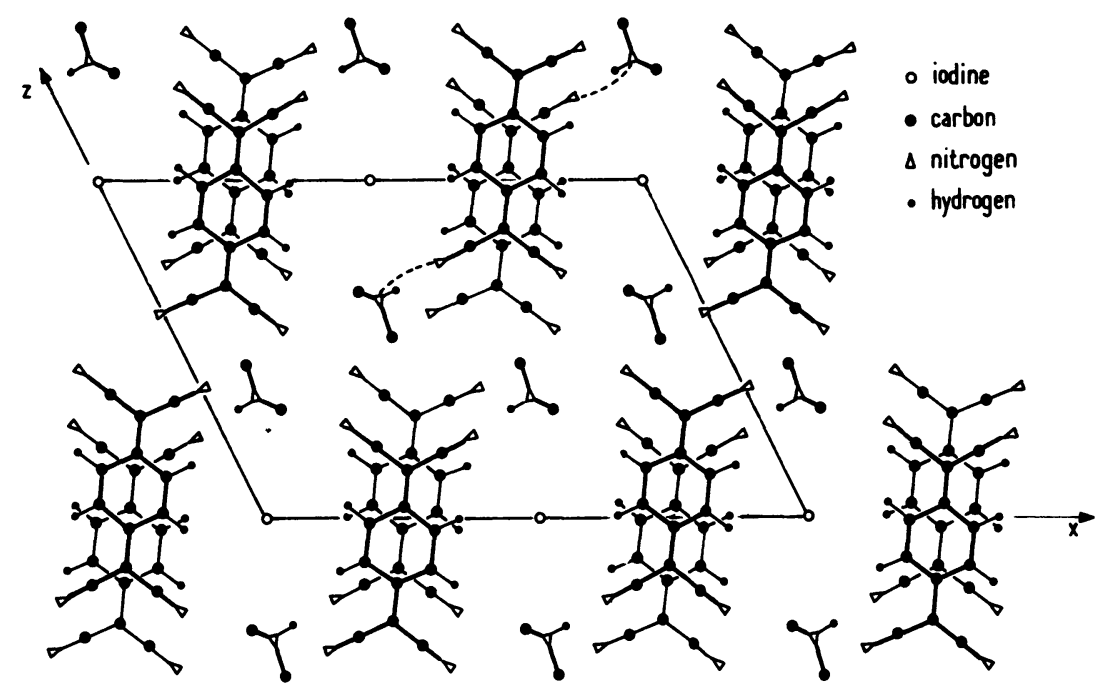

a)

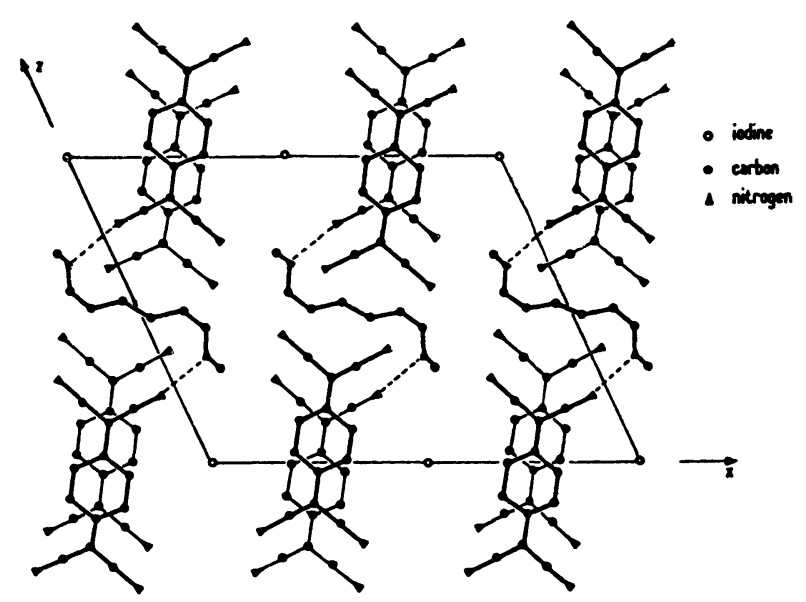

b)

Fig. 1. - Crystal structures at room temperature in the plane orthogonal to the stacking axis of the TMA (1a) and TMHDA (1b) salts (from [6]).

organic reciprocal wave vectors. However, the wave vectors $q_{\mathrm{I}}$ for the TMA and TMHDA salts should be different $[5,8]$.

The structure of IPentDMA-TCNQ-I has been found orthorhombic at $300 \mathrm{~K}$ [9]. However, it seems that this temperature is singular and the structure becomes monoclinic at a slightly lower temperature [10]. In any case, the organization of the TCNQ within a chain is the same as for the other salts. For this compound the structure of the iodine sublattice is very simple being simply periodic with a wave vector $q_{\mathrm{I}}=\left(a^{*}, 2 b^{*} / 3, c^{*}\right)\left(a^{*}, b^{*}, c^{*}\right.$ corresponding to the monoclinic description for which $b^{*}$ is along the chain axis) [9].

The structure of the organic lattice of IPrDMATCNQ-I is also monoclinic [3]. A preliminary X-ray structure indicates that the iodine organization is probably similar to that of IPentDMA salt $\left(q_{\mathrm{I}}=\right.$ $\left.\left(a^{*}, 2 b^{*} / 3, c^{*}\right)\right)[5,8]$.

Finally, it should be noted that only ordered crystals were synthesized up to now, for both TMHDA, IPrDMA and IPentDMA salts.

1.2 LOW TEMPERATURE STRUCTURAL DATA. - The low temperature data concern only the ordered TMA salt for which several studies were reported $[6,9]$. The $T_{\mathrm{c} 1}=150 \mathrm{~K}$ metal-insulator phase transition is associated with the condensation of new spots with the periodicity $q_{0}=\left(a^{*}, b^{*} / 3, c^{*}\right)$ characteristic of a " $2 k_{\mathrm{F}}$ " superstructure. A second transition was shown to affect mainly the iodine sublattice at $T_{\mathrm{c} 2} \simeq$ $80-90 \mathrm{~K}$. This instability was previously discussed with respect to the study of elastic properties [11]. Finally, below $95 \mathrm{~K}$ both a splitting of the organic Bragg spots and a temperature dependence of the $a^{*}$ component of $q_{0}$ are observed. A lock-in of $q_{0}$ with a component close to $a^{*} / 6$ is observed at $T_{\mathrm{c} 3}=65 \mathrm{~K}$. This instability was described as an inc.-comm. transition due to the lock-in of $q_{0}$ at a value commensurate with $q_{\mathrm{I}}$ (TMA) $=\left(2 / 3 a^{*}, 2 / 3 b^{*}, c^{*}\right)$ [4]. It should be 
noted that no structural instability was detected from this detailed structural study for the (D) TMA samples. Up to now a complete structural low temperature study for the other salts of the series is missing.

\section{Physical properties of these compounds.}

2.1 EleCtRICAL CONDUCTIVITY. - The electrical conductivity of the TMA and TMHDA salts has been already published $[12,13,3]$. Contrary to a previous assumption [3], these data always correspond to ordered samples. 'The room temperature values, measured along the needle axis, range between 20 and $40 \Omega^{-1} \mathrm{~cm}^{-1}$ for both compounds. The temperature dependences exhibit either a saturation or a broad maximum at high temperature (Figs. 2 and 3). A sharp maximum of $\mathrm{d} \operatorname{Ln} \rho / \mathrm{d}(1 / T)$ is the signature of the metal-insulator phase transition $\left(T_{\mathrm{c} 1}=150 \mathrm{~K}\right.$ and $118 \mathrm{~K}$ for the TMA and TMHDA salts, respectively). A recent work for the (O) TMA salt is in agreement with these results ; moreover, a kink in the $\sigma(T)$ curve is seen at the second transition 80-85 K [14]. The single crystal resistivity of the (D) TMA salt is given in figure 2 and compared with the behaviour of the (O) samples. The resistivity is always activated; only an increase of $\mathrm{d} \operatorname{Ln} \rho / \mathrm{d}(1 / T)$ is seen below $150 \mathrm{~K}$ with no sign of a critical behaviour. The resistivity temperature dependences of the IPrDMA and IPentDMA salts are close to those of the two first ordered salts (Fig. 3). From these data, the temperatures of the metal-insulator transition were obtained : $T_{\mathrm{c} 1}=136$ and $139 \mathrm{~K}$ respectively (the values of $T_{\mathrm{c}}$ are summarized for the four salts in Table I). Finally, it should be noted that the conductivity of the IPentDMA salt is more pro-

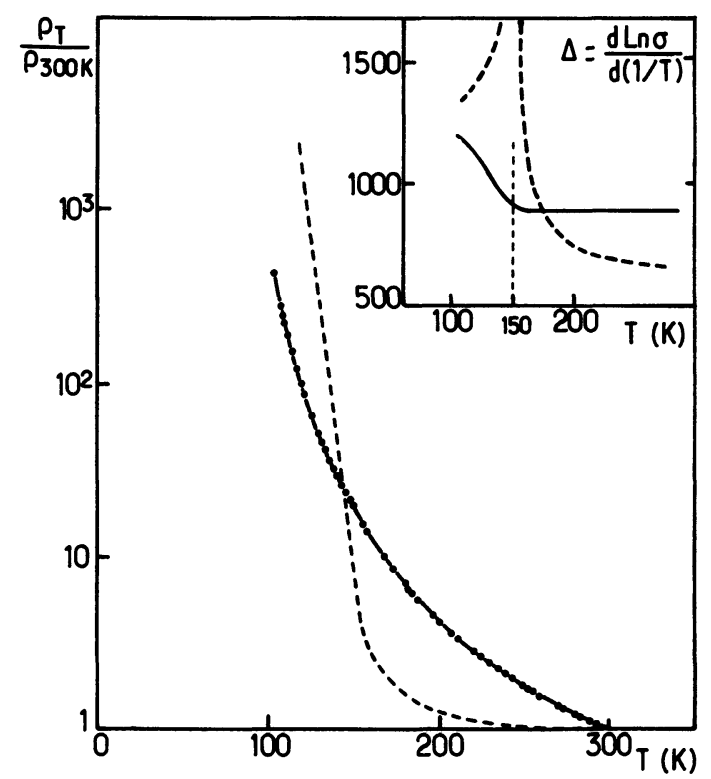

Fig. 2. - Electrical resistivity temperature dependences of $(O)$ and $(D)$ TMA compounds ((O) : dotted lines and (D) : full lines). In insert the logarithmic derivations are given in the vicinity of the phase transition.

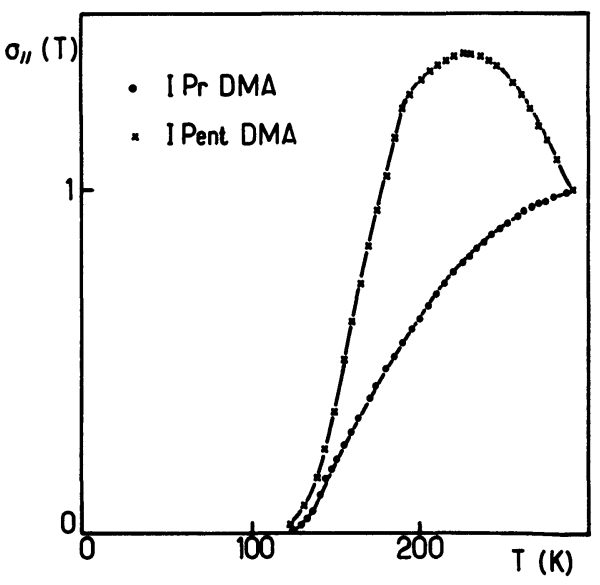

Fig. 3. - Electrical conductivity temperature dependences of the low isomonocation salts (IPrDMA and IPentDMA respectively).

nounced $\left(\sigma_{\max } / \sigma_{300 \mathrm{~K}} \simeq 1.3-1.4\right)$ although $T_{\mathrm{c} 1}$ is not singularly low. This distinctive property might be attributed to slightly different overlaps between the TCNQ chains.

2.2 MAGNETIC MEASUREMENTS. - As previously shown the magnetic susceptibility obtained with the Faraday technique is metal-like for all the compounds in the high temperature range. Then for the ordered samples a drop of the susceptibility is observed at the metal-insulator transition and an activated regime is found below this temperature. Thus, the magnetic susceptibility gives an estimate for the metal-insulator transition temperature which is always in agreement with that of the resistivity measurements [3]. No further information can be gained from this experiment with the exception presented in figure 4 where the behaviours of both (O) and (D) TMA samples are given. The susceptibilities are remarkably similar for both samples above $150 \mathrm{~K}$. Below this temperature a phase transition is only observed for the $(\mathrm{O})$ com-

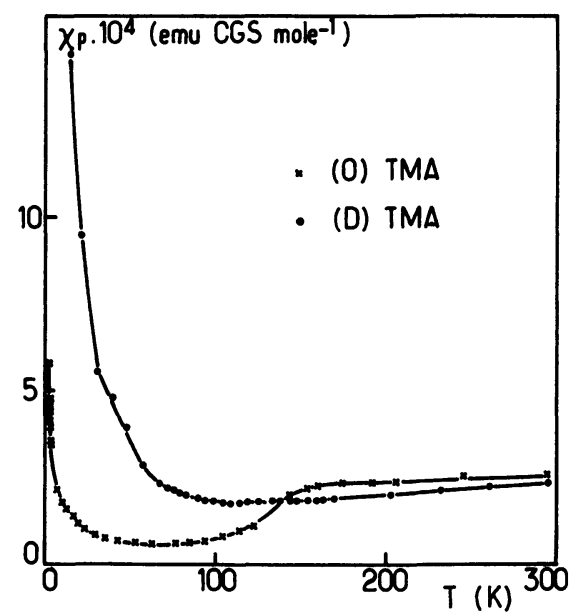

Fig. 4. - Paramagnetic susceptibility temperature dependences of (O) and (D) TMA compounds. 
pound. At low temperature a Curie law is always detected because of the polycrystalline nature of the samples used with this technique. However, this effect is much more pronounced for the (D) salt. We will discuss this point in more detail through EPR results.

2.3 Electronic Paramagnetic ResonanCe. - Only very preliminary and partial results were already published [12]; thus, we will present a detailed study of all the compounds considered. Temperature dependences of the observed resonance line have been carried out between 5 and $300 \mathrm{~K}$ using a standard $\mathrm{X}$-band spectrometer. For each crystal the three main components of the linewidth and $g$-factor second rank tensors have been explored as indicated in figures 5 to 9 .

The behaviour of the two kinds of TMA crystals is given in figures 5 and 6 . For the ordered crystals the EPR linewidth $(\Delta H)$ first decreases in the conducting phase, as usually observed for one-chain compounds. An increase of $\Delta H$ is seen below $T_{\mathrm{c} 1}=150 \mathrm{~K}$, particularly sharp when the static magnetic field $H_{0}$ is along the stacking axis; then each component of the linewidth tensor decreases again at lower temperatures. Nevertheless, an anomaly is observed when $H_{0} / / b$ between 80 and $90 \mathrm{~K}$. The $g$-values are temperature independent as generally observed for the one-chain compounds. Finally, it should be noted that the cor-

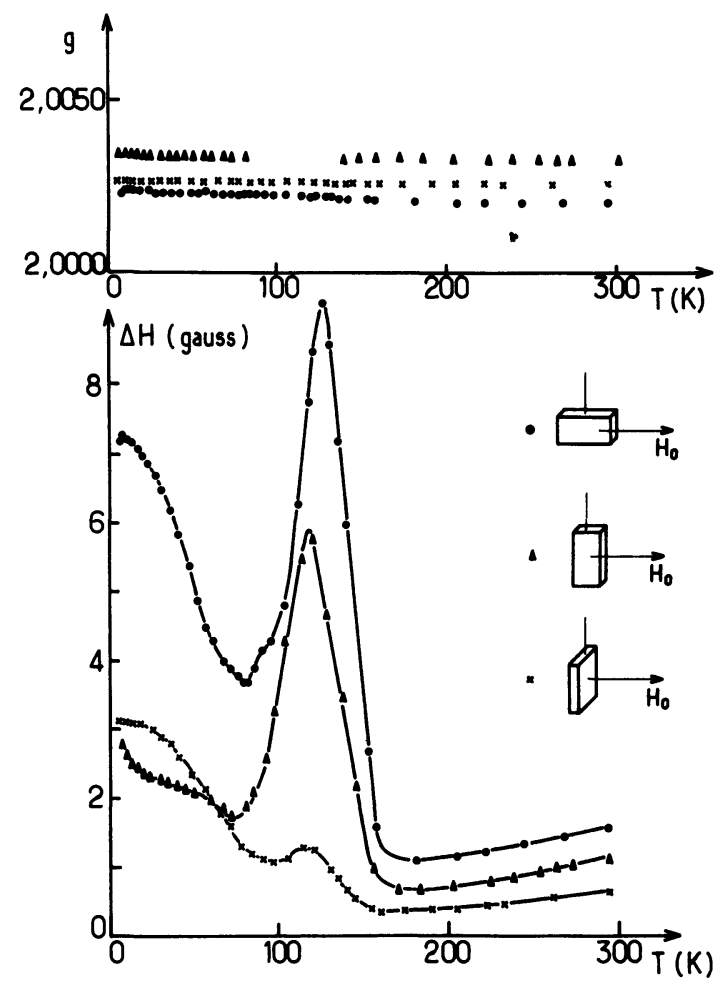

Fig. 5. - Temperature dependences of the linewidth $(\Delta H)$ and $g$-factor principal components for the (O) TMA compound as labelled on the figure (the $b$ crystallographic axis corresponds to the length of the prismatic single crystal).

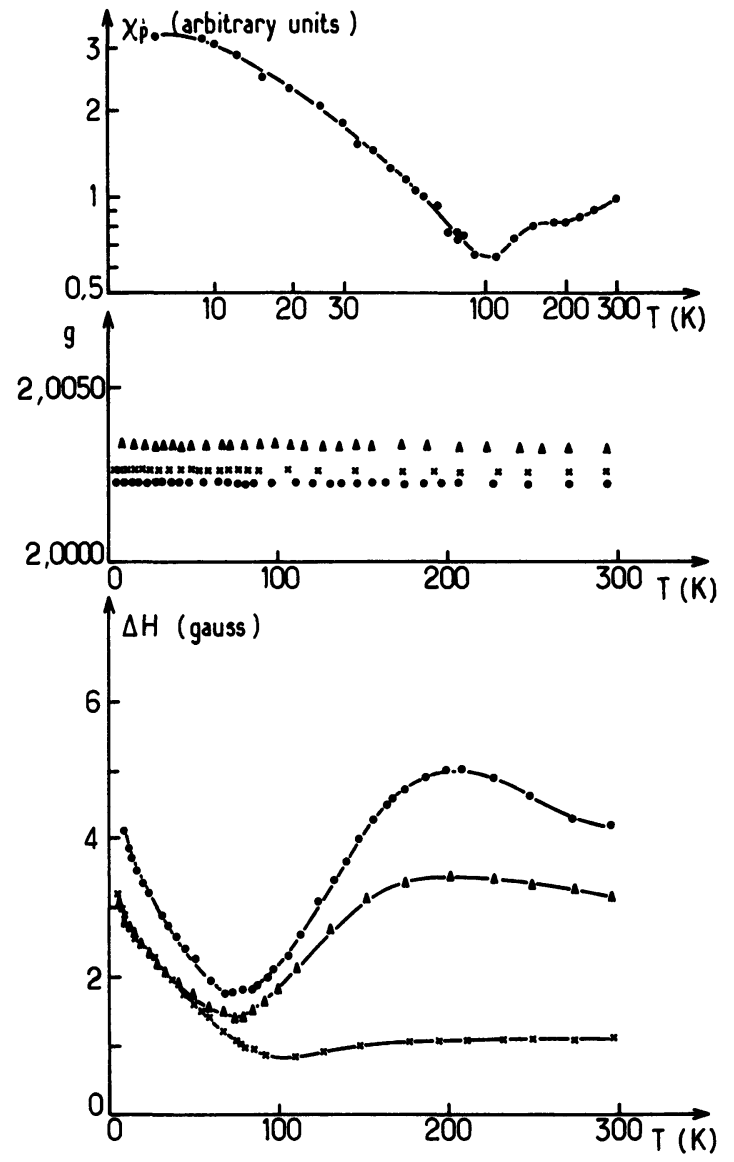

Fig. 6. - Temperature dependences of the linewidth $(\Delta H)$, $g$-factor and integrated line intensity $\left(\chi_{\mathrm{p}}\right.$ in arbitrary units) for the (D) TMA compound. (The different linewidth and $g$-factor components are labelled as already indicated in Fig. 5).

responding susceptibility is in agreement with the static measurement and an activated magnetism is observed below $150 \mathrm{~K}$. However, because this technique needs only one single crystal, no Curie tail was found at low temperature.

The behaviour of the (D) crystals is completely different (Fig. 6). When decreasing the temperature a broad maximum of the linewidth is first observed. The linewidth has a minimum at about $80 \mathrm{~K}$ and increases again at lower temperature. The $g$-values, characteristic of the TCNQ molecule, remain the same as for the ordered salt. The corresponding susceptibility is also given in figure $6:$ no sharp anomaly is detected although $\chi_{p}$ decreases around $150 \mathrm{~K}$. At low temperature the susceptibility increases and follows approximately a $T^{-\alpha}$ law with $\alpha \simeq 0.4$.

The EPR results for the IPrDMA and IPentDMA salts are given in figures 7 and 8 . For the first salt, only partial results were obtained because the EPR spectra were contamined by a narrow line, probably due to a partial degradation of the crystals (the intensity of this line was shown to increase smoothly with the age of the crystals [5]). This is evidence for the metastability of this compound. The same behaviour was found in 

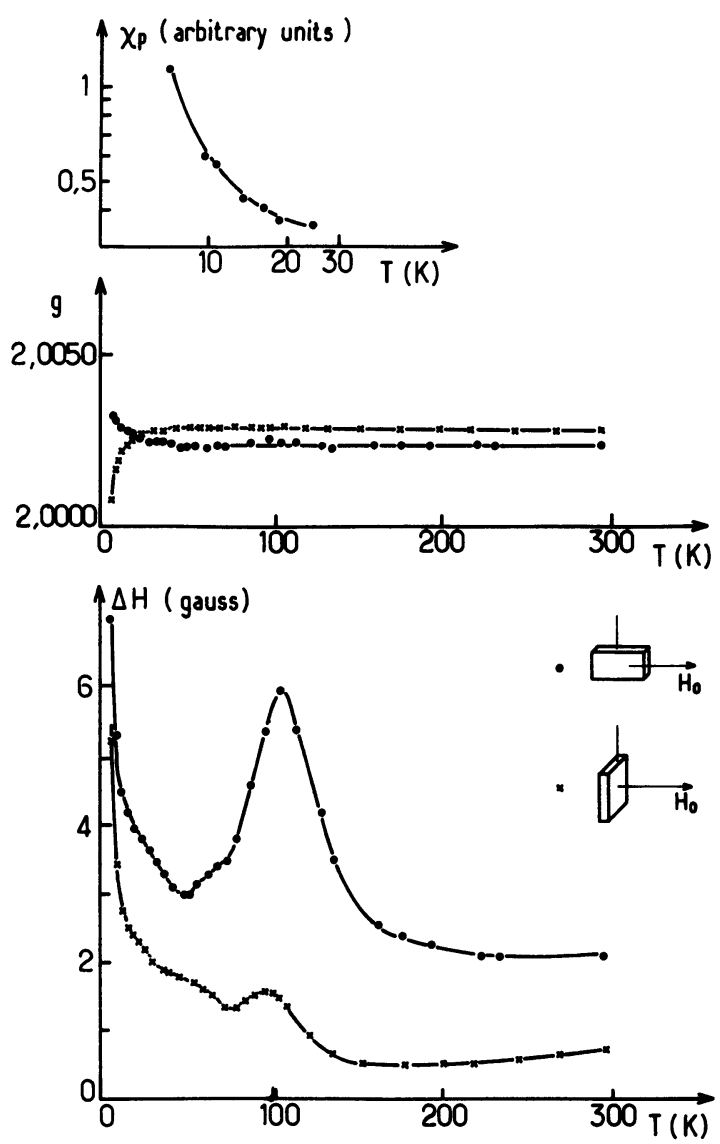

Fig. 7. - Temperature dependences of the linewidth $(\Delta H)$, $g$-factor and integrated line intensity ( $\chi_{\mathrm{p}}$ in arbitrary units) of the IPrDMA compound. (The two linewidth and $g$-factor components are labelled as indicated in Fig. 5.)

several other salts of the series, particularly when the size of the quaternary ammonium cation is large. For the IPrDMA salt, this degradation is probably at the origin of the almost flat temperature dependence of the linewidth in the high temperature phase (as for the d.c. conductivity, see Fig. 3), but does not prevent the EPR investigation below $136 \mathrm{~K}$, and the results given in figure 7 should be considered as intrinsic. The behaviour of the IPrDMA and IPentDMA salts is close to that of the $(\mathrm{O})$ TMA crystals around $100 \mathrm{~K}$ : a maximum of $\Delta H$ is first observed, the amplitude of which decreases with the size of the involved cation. For the IPrDMA a linewidth anomaly is seen between 60 and $80 \mathrm{~K}$, particularly when the static magnetic field is parallel to the stacking axis. The occurrence of such an anomaly in the same temperature range is not clear for the IPentDMA salt. For these two salts, a completely new result is found at low temperature : both the linewidth and the susceptibility diverge and the $g$-factors become temperature dependent. These features are generally the signature of magnetic fluctuations $[15,16]$. We will discuss this point in more detail in the following part of this paper.

The EPR results for the TMHDA salt are given in figure 9. The temperature dependence of the linewidth
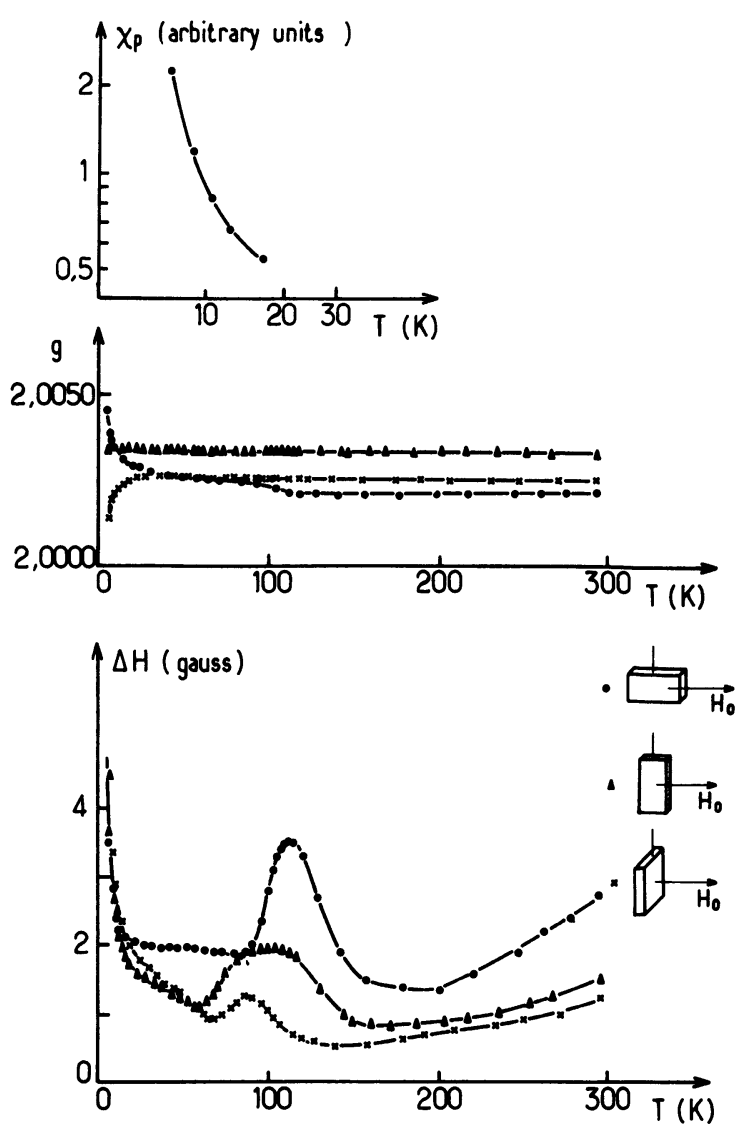

Fig. 8. - Temperature dependences of the linewidth $(\Delta H)$, $g$-factor and integrated line intensity ( $\chi_{\mathrm{p}}$ in arbitrary units) of the IPentDMA compound. (The different linewidth and $g$-factor components are labelled as indicated in Fig. 5).

is different from that of the other salts. A minimum of $\Delta H$ occurs at the phase transition $\left(T_{\mathrm{c} 1}=118 \mathrm{~K}\right)$, particularly when the static magnetic field is along the stacking axis. Then $\Delta H$ increases almost linearly and a second anomaly is seen through a sudden change of slope at $T_{\mathrm{c} 2} \simeq 60-68 \mathrm{~K}$. At lower temperature a behaviour similar to that of the TMA (O) sample is found. Note that the $g$-factors remain always temperature independent. Finally, the susceptibility (not shown in Fig. 9) is activated below $118 \mathrm{~K}$ with no peculiar extra behaviour at very low temperature.

2.4 SPECIFIC HEAT MEASUREMENTS. - From the results shown above, anomalies are detected which may be attributed to the occurrence of phase transitions. To clarify this point we have undertaken specific heat measurements on polycrystalline samples of about 300-500 mg using a differential method; the experimental set-up is described elsewhere [17]. The results are given in figure 10 for the TMA salt (O crystals). Superimposed to a monotonous increase of the specific heat three anomalies are detected respectively at $T=65,90$ and $153 \mathrm{~K}$. This is the usual signature for phase transitions and this measurement confirms, therefore, the existence of the three structural instabi- 


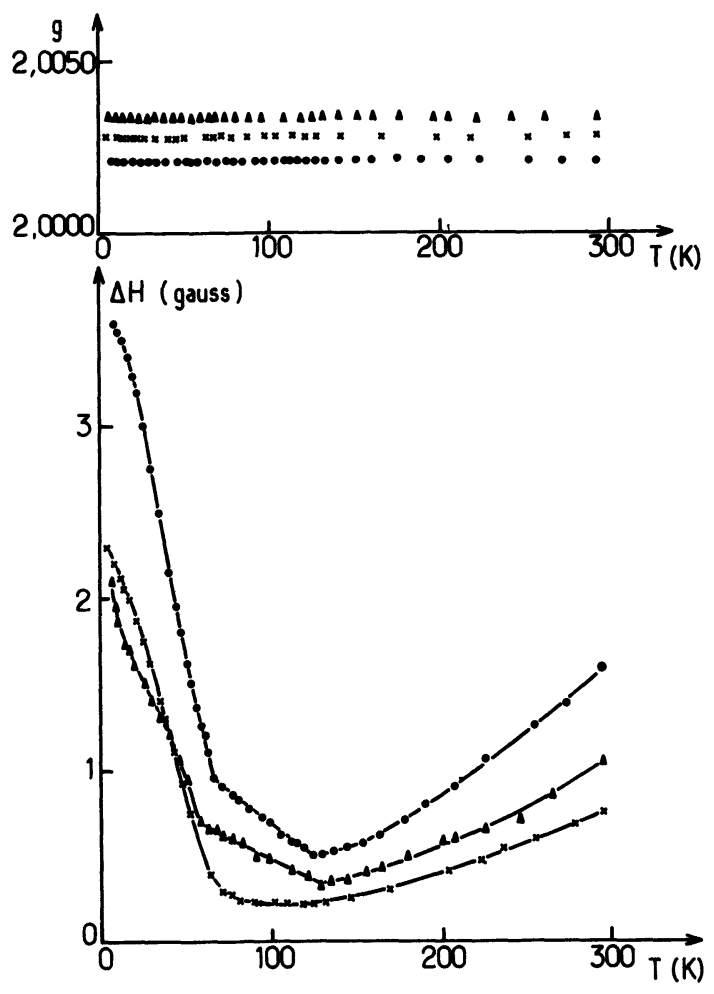

Fig. 9. - Temperature dependences of the linewidth $(\Delta H)$, $g$-factor of TMHDA compound. (The different linewidth and $g$-factor components are labelled as indicated in Fig. 5.)

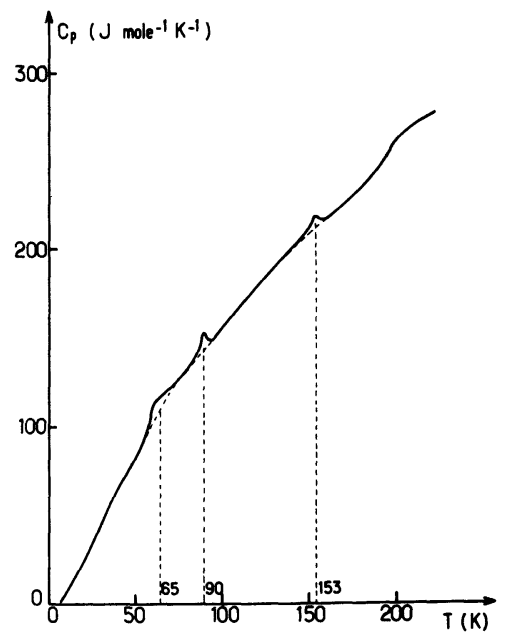

Fig. 10. - Specific heat temperature dependence of $(O)$ TMA salt.

lities described in part I. The weakness of the observed bumps does not allow a discussion on the order of these instabilities. However, the $90 \mathrm{~K}$ phase transition appears sharper in agreement with its already reported first order character $[11,14]$. In addition to these three anomalies a very broad bump of the specific heat is observed above $200 \mathrm{~K}$. This part of the curve, although always observed, is not completely reproducible. In connection with other results on organic materials [18] we suggest that this anomaly is related to an iodine lattice effect which was observed with X-ray diffraction above $150 \mathrm{~K}$ [4].

Concerning the other salts, two sets of experiments have been carried out on IPrDMA and TMHDA salts. Probably because of the iodine chemical instability the data on two different batches of the IPrDMA salt were poorly reproducible. A specific heat anomaly is observed at about $139 \mathrm{~K}$ in both experiments, this anomaly corresponds to the metal-insulator phase transition (see Table I). Besides, a bump has been detected once around 70-100 K; from this result we cannot exclude the presence of a second phase transition in this temperature range. Other preliminary results have been obtained for the TMHDA compound. Three anomalies are observed at about 60-80 and $120 \mathrm{~K}$. Thus, two other instabilities seem to exist below the metal-insulator phase transition for this salt, exactly as observed for the TMA compound (see Table I).

\section{Discussion.}

3.1 The TMA SALT. - Since a detailed study of the low temperature structural properties of TMA-TCNQI was recently published [6,4], we will begin the discussion with this compound.

For the (D) type crystals no sign of structural distortion was found [4]. This is in agreement with our results : no sharp anomaly was seen in both the electrical and EPR results. However, a decrease of the EPR magnetic susceptibility and an increase of $\mathrm{d} L \mathrm{n} \rho / \mathrm{d}(1 / T)$ are seen around $150 \mathrm{~K}$. We suggest that these observations are related to the growth of short-range order of the distortion which occurs at $150 \mathrm{~K}$ in the $(\mathrm{O})$ crystals. Nevertheless, the behaviour of the (D) sample is dominated by the disorder of the iodine chains as suggested by the $T^{-\alpha}$ low-temperature dependence of the susceptibility, which is characteristic of disordered solids [12].

For the $(\mathrm{O})$ crystals three phase transitions were reported $[3,19,20]$. The origin of these instabilities was recently discussed through low temperature structural results [4]. The $150 \mathrm{~K}$ instability has been shown to be associated with the condensation of a superstructure with the wave vector $q_{0}=\left(a^{*}, b^{*} / 3, c^{*}\right)$. Because of the given charge transfer ( $2 / 3$ electron per TCNQ) the periodicity of the superstructure allows a reduction of the electronic energy (the $b^{*}$ component of $q_{0}$ is exactly $2 k_{\mathrm{F}}$ ) and the distortion was described as a Peierls-like distortion. Furthermore, in these ordered crystals the organization of the iodine lattice is described in first approximation as a periodic array of charges with the wave vector $q_{1}=\left(2 a^{*} / 3,2 b^{*} / 3, c^{*}\right)$ and the $a^{*}$ component of $q_{1}$ prevents any strong coupling between the $\mathrm{I}_{3}^{-}$chains and the organic superstructure. This coupling is realized at $T_{\mathrm{c} 3} \simeq 65 \mathrm{~K}$ because the $a^{*}$ component of $q_{0}$ becomes temperature dependent 
below $95 \mathrm{~K}$ to reach a value close to $a^{*} / 6$ at $65 \mathrm{~K}$. This new component is commensurate with that of the iodine wave vector $q_{\mathrm{I}}$ and the $65 \mathrm{~K}$ phase transition has been described as a commensurate-incommensurate phase transition [5]. Finally, a structural phase transition takes place at $T_{\mathrm{c} 2} \simeq 82-90 \mathrm{~K}$, changing mainly the iodine organization. Because of the lack of structural data, no detailed analysis of this distortion is possible.

For these $(\mathrm{O})$ crystals the three phase transitions are clearly seen from the specific heat data. This measurement is made while increasing the temperature and the location of these instabilities is probably slightly overestimated. This is particularly true for the $T_{\mathrm{c} 2}$ structural distortion which has been shown to be a first order instability [4]. Thus, the temperature observed for the distortion is in good agreement with previous studies $[11,14]$.

The other physical properties described in this paper also allow the detection of the phase transitions. As shown previously [3], both the EPR and static magnetic susceptibilities and the electrical conductivity present an anomaly at the $150 \mathrm{~K}$ metal-insulator transition (see Figs. 2 and 4). For this compound the EPR linewidth increases below $150 \mathrm{~K}$ (see Fig. 5), and presents also a sharp minimum around $82 \mathrm{~K}$, probably associated with the $T_{\mathrm{c} 2}$ distortion. A comparison of the EPR data of the different samples will be given in more detail in the following.

3.2 COMParison WITH THE OTHER SALTS. - The discussion about the other salts of the series has to be more speculative because of the lack of low temperature structural data. However, a comparison of the physical properties of the different compounds is fruitful, mainly with the help of the EPR and specific heat results.

In a previous study, the metal-insulator transition temperature $\left(T_{\mathrm{c} 1}\right)$ was shown to depend strongly on the size of the cation [3]. This result was explained as a consequence of the decrease of the interchain couplings $\left(W_{\perp}\right)$ when the $c$-lattice parameter is increased as a consequence of the increase in cation size. In agreement with this point of view a single curve $T_{\mathrm{c} 1}\left(W_{\perp}\right)$ is obtained for most of the compounds. Such a result is rather unexpected if one realizes that the role of the counterions is completely ignored in this picture. It should be noted that contrary to the assumption made in [3], the salts described for this study, including the TMA salt, are ordered samples.

This simplified description fails however to explain several experimental results. On one hand, an important decrease of $T_{\mathrm{c} 1}$ (see Table I) is observed when TMA is substituted by the TMHDA cation in agreement with the change of $W_{\perp}$ and these two compounds belong to the $T_{\mathrm{c} 1}\left(W_{\perp}\right)$ curve. On the other hand, $T_{c 1}$ is almost the same for the IPrDMA and IPentDMA compounds although the size of the anions is noticeably different. A calculation of $W_{\perp}$ reveals that these two salts do not belong to the $T_{\mathrm{c} 1}\left(W_{\perp}\right)$ curve, the observed transition temperatures being too high [5]. Moreover, the EPR results presented above clearly show the role of the counterions (see for example the temperature dependence of the EPR linewidth below $T_{\mathrm{c} 1}$ ). We will discuss these two points in the following.

3.2.1 Low temperature instabilities. - The first point to discuss is the relevance of the simplified description of reference [3]. As previously mentioned for the TMA salt, no strong coupling exists between the organic superstructure and the iodine lattice at $150 \mathrm{~K}$. The reason for this is the non integer $a^{*}$ component of the iodine wave vector. Preliminary results also indicate that the iodine wave vector for the TMHDA salt has non integer components $[5,8]$. For these two salts the iodine sublattice will not play an important role for the establishment of the long-range order at $T_{c 1}$. Because the driving force of the distortion is not expected to be due to the cations [4], for these two compounds the temperature of the metal-insulator phase transition is not mainly determined by the counterions and the simplified description proposed in reference [3] may be relevant.

On the other hand, for the IPrDMA and IPentDMA salts the wave vector describing the iodine sublattice is $q_{\mathrm{I}}=\left(a^{*}, 2 b^{*} / 3, c^{*}\right)[9,8]$. The structure of the TCNQ lattice being similar to that of the TMA salt, the wave vector of the superstructure is always expected to be $q_{0}=\left(a^{*}, b^{*} / 3, c^{*}\right)$. In this case there will be a strong coupling between the $\mathrm{I}_{3}^{-}$chains and the organic superstructure, since the relation $q_{\mathrm{I}}=2 q_{0}+G$ (where $G$ is a reciprocal wave vector of the organic lattice) is satisfied. This coupling plays an important role in determining $T_{\mathrm{c} 1}$, which is no more strongly dependent on $W_{\perp}$. As expected, the observed value of $T_{\mathrm{c}}$ is larger than the estimate from the relation $T_{\mathrm{c} 1}\left(W_{\perp}\right)$ of reference [3].

The possible occurrence of a commensurate-incommensurate phase transition, as observed for the TMA salt $\left(T_{\mathrm{c} 3}=65 \mathrm{~K}\right)$, may also be predicted for the TMHDA salt. The existence of such an instability needs a weak coupling between the iodine lattice and the TCNQ distortion at the metal-insulator phase transition. The lock-in between the two charge distributions may be realized at lower temperature as suggested by the occurrence of a phase transition around $T_{\mathrm{c} 3}=60 \mathrm{~K}$. Furthermore, a structural distortion probably due a reorganization of the iodine sublattice is found for the TMA salt at $T_{\mathrm{c} 2} \simeq 85 \mathrm{~K}$. If the driving force for the instability involves mainly the elastic energy of the lattice, this phase transition may be found for all the ordered crystals whatever the iodine sublattice : this assertion may be valid for TMHDA $\left(T_{\mathrm{c} 2} \simeq 80 \mathrm{~K}\right)$.

Now for the two other salts, IPrDMA and IPentDMA, no temperature dependence of $q_{0}$ at low temperature is expected, as explained in the previous paragraph, since a commensurate distortion is established at $T_{\mathrm{c} 1}$. Nevertheless, the existence of a struc- 
tural transition cannot be excluded in the light of the present data, as possibly suggested by the specific heat and EPR results.

In summary, the temperatures of the observed phase transitions are gathered in table $I$.

3.2.2 Low temperature magnetic behaviour. - Although no general firm theory exists for the EPR behaviour of the quasi-1d organic conductors, the results given in 2.3 are relevant for a comparison of the different salts.

As we will discuss hereafter, taking into account the counterions is probably important in explaining some of these results. For the three salts derived from a monocation, a maximum of the EPR linewidth is observed which is particularly pronounced when the static magnetic field is applied along the stacking axis. This behaviour is found whatever the organization of the iodine chains; the amplitude of the maximum is decreasing with the cation size. A partial result obtained for the heptyldimethylammonium salt indicates that only a small maximum exists for this compound made with a large monocation [5]. This result supports the rule mentioned above which may be quite general. The occurrence of a maximum has to be associated with the metal-insulator phase transition : below $T_{\mathrm{c} 1}$ a periodic lattice distortion appears on the TCNQ chains and an inhomogeneous broadening effect is expected, as already found in other compounds [21]. Such a situation appears to be encountered for these ternary salts as far as the monocations are involved. The effect is weaker and no maximum is found for the dication salts like the TMHDA compound. The origin of the difference between the monocation and dication salts may be a consequence of the structure of these compounds (see Fig. 1) : the $150 \mathrm{~K}$ distortion for the (O) TMA crystals was shown to involve mainly displacements of the TCNQ molecules in the $a$ direction [6]. Because strong hydrogen bonds exist between TCNQ molecules and the cations, the TMA ions are probably also displaced when the organic chains are distorted (to preserve the length of the $\mathrm{H}$ bonds). The situation is probably the same for the other monocation salts, although the displacement of the anions is expected to be more difficult when their size increases. For the dications salts, however, each cation is interacting with two TCNQ chains and the displacement is probably smaller, in agreement with preliminary $\mathrm{X}$ ray diffuse scattering observations [8].

A distinctive observation is made for the IPrDMA and IPentDMA salts at very low temperature, as the $g$-factors, linewidth, and magnetic susceptibility become strongly temperature dependent.

This behaviour is usually the signature of antiferromagnetic fluctuations $[15,16]$ which may announce the condensation of a long range order of the spins at a lower temperature $[15,22]$. Such a phase transition is, however, unexpected for the studied compounds since a gap is opened below 136-139 $\mathrm{K}$ in the spin excitations spectrum. To reconcile these arguments one may speculate that magnetic defects are present in the TCNQ chains. Considering a 1d correlated electronic system, it is found that magnetic configurations of low energy may be generated [23]. As shown in figure 11, these configurations may be introduced as " defects " without breaking the long-range order of the structural distortion of the chains. It is clear from figure 11 that a defect contains two magnetic units (with an odd number of spins) and that these defects may be at the origin of the observed magnetic behaviour. Since the low temperature structure of the IPrDMA or IPentDMA is not known, it is presently impossible to discuss why the occurrence of these defects may be found for these two salts. Furthermore, the occurrence of a short-range magnetic order although suggested by the EPR data remains controversial. Magnetic data at very low temperature would probably be useful to clarify this point.

3.3 ConClusion. - We have investigated in detail the physical properties of four compounds of the series of ternary TCNQ salts. In connection with recent structural results the low temperature behaviours of these salts have been compared. A strong interconnection was found between the counterions and the nature of the electronic states. In the disordered TMA sample the random potential due to the iodine inhibits the condensation of new low temperature ground states. For the ordered crystals the organization of the $\mathrm{I}_{3}^{-}$chains is a relevant parameter which determines the strength of the coupling of their sublat-

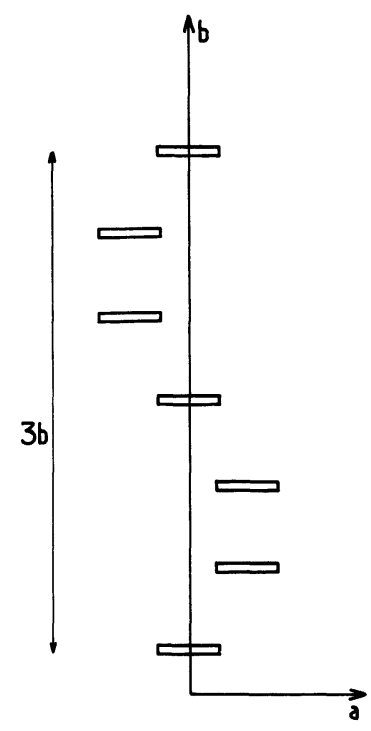

(a)

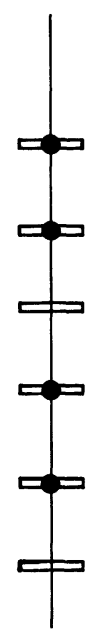

(b)

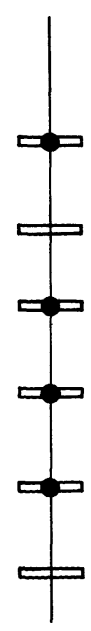

(c)
Fig. 11. - a) Approximate TCNQ organization at $100 \mathrm{~K}$ for (O) TMA-TCNQ-I (the molecule displacements are amplified for clarity).

b) Electronic localization in this low $T$ state (schematic).

c) Possible topological defect of this electronic configuration. Note that the long-range structural order is not destroyed since the size of the defect is equal to $3 b$. 
tice with the instabilities for the TCNQ chains. The most striking consequence of this coupling is the possible occurrence of a commensurate-incommensurate phase transition as observed for the TMA salt at $65 \mathrm{~K}$. Moreover, the cations also play an important role, probably determining the type of molecular displacements which occur below the metal-insulator phase transition.

When examined in detail, the physical properties of these ternary salts appear rather complex but very interesting. One of the most surprising results is the low temperature magnetic behaviour of the IPrDMA and IPentDMA salts, which can only be explained if magnetic units are introduced on the chains. Moreover, the EPR data suggest the occurrence of a growing short-range magnetic order when the temperature is reduced. Further experiments at lower temperatures would certainly be useful to probe the possible occurrence of the condensation of a long range magnetic order.

\section{Acknowledgments.}

It is a pleasure to acknowledge B. Gallois and J. P. Pouget for numerous discussions on the structural properties of these compounds.

\section{References}

[1] For a recent summary see for example : Proceedings of the international "Les Arcs" conference, $J$. Physique Colloq. 44 (1983) C3.

[2] Dupuis, P., Flandrois, S., Delhaes, P., Coulon, C., J. Chem. Soc. Chem. Comm. 8 (1978) 328.

[3] Coulon, C., Flandrois, S., Delhaes, P., Hauw, C. Dupuis, P., Phys. Rev. B 23 (1981) 2850.

[4] Gallois, B., Coulon, C., Pouget, J. P., Dupuis, P. (to be published).

[5] Coulon, C., Ph.D. Thesis Bordeaux University (1982).

[6] For a review see : Gallois, B., Gaultier, J., Granier, T., Ayrolles, R., Filhol, A. (to be published).

[7] Coppens, P., Leung, P., Murphy, K. E., Tilborg, P. R., Epstein, A. J., Miller, I. S., Mol. Cryst. Liq. Cryst. 61 (1980) 1.

[8] Coulon, C., Pouget, J. P. (Unpublished work).

[9] Chasseau, D., Filhol, A., Gaultier, J., Hauw, C., Steiger, W., Chem. Scr. 17 (1981) 97.

[10] Gallois, B. (Private communication).

[11] Brill, J. W., Epstein, A. J., Miller, J. S., Phys. Rev. B 20 (1979) 681.

[12] Cougrand, A., Flandrois, S., Delhaes, P., Hauw, C., Dupuis, P., Mol. Cryst. Liq. Cryst. 32 (1971) 165.

[13] Abkowitz, M. A., Epstein, A. J., Griffiths, G. H., Miller, J. S., Slade, M. L., J. Am. Soc. 99 (1977) 5304.
[14] Ikari, T., Jandl, S., Aubin, M., Truong, K. D., Phys. Rev. B 28 (1983) 3859 and

Bandrauk, A. D., Truong, K. D., Carlone, C., JANDL, S., in reference [1], p. 1473.

[15] Coulon, C., Maaroufi, A., Amiell, J., Dupart, E., Flandrois, S., Delhaes, P., Morand, J. P., Phys. Rev. B 23 (1982) 2850.

[16] Bozler, H. M., Gould, C. M., Clark, N. G., Phys. Rev. Lett. 45 (1980) 1303.

[17] Lagnier, R., Pierre, J. and Mortimer, M. J., Cryogenics 17 (1980) 349.

[18] Ayache, C. and Torrance, J. B., Solid State Commun. 47 (1983) 789.

[19] Filhol, A., Gallois, B., Laugier, J., Dupuis, P., Coulon, C., Mol. Cryst. Liq. Cryst. 84 (1982) 17.

[20] Granier, T., Ayrolles, R., J. Physique Lett. 43 (1982) L-285.

[21] JACOBS, I. S., BRAY, J. W., HART, H. R., INTERRANTE, L. V., Kasper, J. S., Watking, D. G., Prober, B. E. and Bonner, J. C., Phys. Rev. B 14 (1976) 3036.

[22] Korving, W. H., Hijmans, T. W., Brom, H. B., Oostra, S., Sawatzky, G. A. and Kommandeur, J., J. Physique Colloq. 44 (1983) C3-1425.

[23] Mazumdar, S., Dixit, S. N., Bloch, A. N., Phys. Rev. B 30 (1984) 4842. 